\title{
Cellular Mechanisms Underlying the Anxiolytic Effect of Low Doses of Peripheral $\Delta^{9}$-Tetrahydrocannabinol in Rats
}

\author{
Tiziana Rubino*, I,3, Mariaelvina Sala ${ }^{2,3}$, Daniela Viganò', Daniela Braida ${ }^{2}$, Chiara Castiglioni', \\ Valeria Limonta ${ }^{2}$, Cinzia Guidali', Natalia Realini' ${ }^{1}$ and Daniela Parolaro' \\ 'DBSF, Pharmacology Section and Center of Neuroscience, University of Insubria, Busto Arsizio, Varese, Italy; ${ }^{2}$ Department of Pharmacology, \\ Chemotherapy and Medical Toxicology, Faculty of Sciences, University of Milan, Milan, Italy
}

\begin{abstract}
We investigated the effect of low doses of intraperitoneal $\Delta^{9}$-tetrahydrocannabinol (THC) on anxiety behavior in rats using the elevated plus maze (EPM). An anxiolytic effect was obtained in a range of doses between 0.075 and $1.5 \mathrm{mg} / \mathrm{kg}$, the 0.75 dose being the most effective. Pretreatment with the CBI receptor antagonist AM25 I fully reversed THC's effect, suggesting CBI receptors were involved. In order to elucidate the neuroanatomical substrates underlying the effect of the maximal effective dose of THC, we investigated cFos expression in anxiety-related brain regions (prefrontal cortex, nucleus accumbens, amygdala, and hippocampus) of rats exposed to the EPM. THC significantly lowered the amount of cFos in prefrontal cortex and amygdala without affecting the other cerebral areas. As there is increasing evidence that CREB function regulates anxiety-like behavior in rats, the second biochemical parameter we measured was phosphorylated CREB in the same brain areas. Rats treated with THC showed a significant increase in CREB activation in the prefrontal cortex and hippocampus. In the prefrontal cortex this increased activation was linked to an increase in ERK activation, whereas in the hippocampus there was a drop in the activity of CAMKII, a kinase with inhibitory effect on CREB activation. All these effects were reversed by $\mathrm{AM} 25 \mathrm{I}$ pretreatment, suggesting that stimulation of $\mathrm{CB}$ I receptors is fundamental for triggering the biochemical events. Our results suggest that the stimulation of these receptors in the prefrontal cortex, amygdala, and hippocampus with the subsequent activation of different signaling pathways is the first event underlying the effects of cannabinoids on anxious states. Neuropsychopharmacology (2007) 32, 2036-2045; doi: I 0.I 038/sj.npp. I 30 I330; published online 7 February 2007
\end{abstract}

Keywords: THC; anxiety; cFos; brain regions; CREB; cellular mechanisms

\section{INTRODUCTION}

The role of cannabinoids in the modulation of anxious states is still a matter of controversy. In humans, cannabis use could promote either relaxation and euphoria, thus relieving anxious state, or anxiety and panic attacks, depending on subjects, and, within the same subject, on the emotional state prior the use (Iversen, 2003). Similarly, animal experiments with different cannabinoid agonists revealed both anxiolytic-like and anxiogenic-like responses (for a review, see Viveros et al, 2005) depending on the doses and the familiarity of the environment. Natural and synthetic cannabinoids seem to display a dose-dependent biphasic profile in rats and mice when using standard anxiety models. Low doses of these compounds produced anxiolytic-like responses (Valjent et al, 2002; Berrendero

* Correspondence: Dr T Rubino, DBSF, Pharmacology Section and Center of Neuroscience, University of Insubria, via A. da Giussano 10 , 21052 Busto Arsizio, Varese, Italy, Tel: + 390331 3394I6, Fax: + 39 033। 339459, E-mail: tiziana.rubino@uninsubria.it

${ }^{3}$ These two authors contributed equally to this work

Received I0 April 2006; revised 21 November 2006; accepted II December 2006 and Maldonado, 2002; Genn et al, 2004; Marco et al, 2004; Rodriguez de Fonseca et al, 1996), whereas higher doses resulted in anxiogenic-like effects in several models of anxiety (Onaivi et al, 1990; Arevalo et al, 2001; Marin et al, 2003; Genn et al, 2004; Marco et al, 2004). Recent data obtained with pharmacological agents that enhance the endocannabinoid tone join this complex picture suggesting that anandamide too has a role in the regulation of anxiety states (Bortolato et al, 2006; Kathuria et al, 2003). Peripheral injection of the endocannabinoid transport inhibitor AM404 or the active inhibitor of fatty acid amide hydrolase URB597 produced anxiolytic-like effects in different rat models of anxiety. These effects were accompanied by an increase in the brain level of anandamide and were prevented by $\mathrm{CB} 1$ receptor blockade.

Besides the confusing picture from behavioral experiments, the mechanisms of the effects of cannabinoids on anxiety-related responses still need further investigation. Although it has been proposed that the corticotropinreleasing hormone (CRH) system as well as CCK and serotonin (see for review Viveros et al, 2005) may participate in cannabinoids' effects on anxiety behavior, the intracellular pathways activated by the stimulation of CB1 receptors, involved too, are still unknown. 
The present study investigated the effect of low doses of THC on anxiety behavior in rats, measured in the EPM. To clarify the neuroanatomical substrates of the effect of intraperitoneally injected THC, we also investigated cFos expression in anxiety-related brain regions (prefrontal cortex, nucleus accumbens, amygdala, and hippocampus) of rats exposed to the EPM. Assessment of cFos expression is the most widely used functional anatomical mapping tool to identify cells and extended circuitries that become activated in response to various stimuli including anxiogenic environment (Kovacs, 1998). The second biochemical parameter we measured was phosphorylated CREB (ie activated CREB) in nuclear extracts from the prefrontal cortex, nucleus accumbens, amygdala, and hippocampus of rats treated with THC and exposed to EPM, as CREB function probably regulates anxiety-like behavior in rats (Barrot et al, 2002; Pandey, 2003). In the positive brain regions we then investigated the CB1-dependent intracellular pathways activated by THC that might account for the CREB modulation. There are numerous protein kinases that regulate CREB activity differently: protein kinase A (PKA) and ERK-activated ribosomal S6 kinases (RSKs) phosphorylate CREB and lead to activation of gene transcription (Carlezon et al, 2005); by contrast, $\mathrm{Ca}^{2+} /$ calmodulindependent kinase (CAMK) II reduces CREB-mediated gene transcription (Wu and McMurray, 2001). In vivo stimulation of CB1 receptors can affect PKA and ERK activity (Rubino et al, 2000, 2004; Valjent et al, 2001; Hampson et al, 1995), but there is no evidence of their ability to alter CAMK II in vivo, although the inhibition of voltage-gated calcium channels is an established cannabinoid transduction pathway (Howlett and Mukhopadhyay, 2000). We therefore investigated the activity of PKA, ERK, and CAMKII.

\section{METHODS}

\section{Subjects}

All the experimental procedures followed the guidelines of the Italian Council on Animal Care, and were approved by the Italian Government decree No. 33/2004. All efforts were made to minimize the number of animals used and their suffering.

At the beginning of the experiment, maze-naïve male Sprague-Dawley rats (Charles-River, Calco, Italy), weighing $150-175 \mathrm{~g}$, were housed in cages (10 per cage) in a climatically controlled colony room under a 12-h lightdark cycle (lights on at 0800 hours). Food and water were continuously available and each animal was handled daily during the 7 days preceding the experiment. The day before the experiment animals were individually housed and randomly assigned to each experimental group (10 rats per group). Rats were used only once.

\section{Drugs and Treatments}

The following drugs were used: THC (kindly supplied by GW Pharma, Salisbury, England), in a range of doses between 0.015 and $3 \mathrm{mg} / \mathrm{kg}$ and dissolved in cremophor, ethanol, and saline $(1: 1: 18)$. AM251 $(3 \mathrm{mg} / \mathrm{kg})$ was obtained from Sigma-Aldrich MO (St Louis, USA), and dissolved in DMSO 10\% and saline 90\%. THC was given acutely i.p. 30 min before, whereas AM251 was given $40 \mathrm{~min}$ before the test. SL327 (Tocris, Bristol, UK) was dissolved in $30 \%$ DMSO. The doses of the drugs were calculated as salt.

\section{Surgery}

For intracerebral surgery, rats were anesthetized with chloral hydrate $(400 \mathrm{mg} / \mathrm{kg}$ i.p.) and guide cannulae for prefrontal cortex microinjections were stereotaxically implanted unilaterally and cemented to the skull (coordinates with references to bregma according to stereotaxic atlas of Paxinos and Watson: prefrontal cortex AP $+2.7 \mathrm{~mm}$, ML $0.8 \mathrm{~mm}$, DV $3 \mathrm{~mm}$ ). Immediately after surgery, rats were given an injection of amoxicilline $(20 \mathrm{mg} / \mathrm{kg})$ and then allowed 7 days to recover from surgery before any behavioral testing.

\section{Behavioral Studies}

Elevated plus maze. The elevated plus maze (EPM) consisted of two opposite open arms $(50 \times 10 \mathrm{~cm})$ and two enclosed arms $(50 \times 10 \times 40 \mathrm{~cm})$ extended from a common central platform $(10 \times 10 \mathrm{~cm})$ based on a design validated by Lister (1987). The entire apparatus was constructed of white-painted wood, elevated to a height of $50 \mathrm{~cm}$ above floor level and placed in the center of a small room that was lit with fluorescent lights.

Upon completion of injection made in the colony room, animals were transported to plus-maze laboratory to facilitate adaptation to novel surroundings for $30 \mathrm{~min}$. Then, rats were placed individually onto the center of the apparatus facing an open arm, and the time spent on and entries onto each arm were detected for $5 \mathrm{~min}$. The maze was wiped clean with water and dried after each trial. All experimental sessions were conducted during the morning time of the light cycle (1000-1400 hours). An arm entry was recorded when all four paws of the rat were in the arm. The number of open- and closed-arm entries and the time spent in each arm were recorded. Data were expressed as the percentage (open or closed time/total time $\times 100$; open or closed entries/total entries $\times 100$ ). The percentage of time spent in the open arms and the percentage of open-arm entries were used as measures of anxiety (Hogg, 1996).

Spontaneous motor activity. Spontaneous motor activity was evaluated as previously described () in an activity cage $(43 \times 43 \times 32 \mathrm{~cm})$ (Ugo Basile, Varese, Italy), placed in a sound-attenuating room. The cage was fitted with two parallel horizontal and vertical infrared beams located 2 and $6 \mathrm{~cm}$ from the floor. Cumulative horizontal and vertical movement counts were recorded $30 \mathrm{~min}$ after treatment with vehicle or THC for $15 \mathrm{~min}$.

\section{Biochemical Studies}

After the EPM session, rats were killed and brains quickly removed. In these studies, a group of animals handled as the ones exposed to EPM but not tested (naïve) was also present to check the level of the biochemical parameters in basal conditions. The cerebral areas (prefrontal cortex, nucleus accumbens, amygdala, and hippocampus) were obtained within a few minutes by regional dissection on ice according 
to Heffner et al (1980), and immediately frozen in liquid nitrogen and stored at $-80^{\circ} \mathrm{C}$ until used.

Tissue preparation. Each brain region was homogenized in an appropriate volume of ice-cold Buffer A (10 mM Hepes $\mathrm{pH} 7.5,1.5 \mathrm{mM} \mathrm{MgCl}_{2}, 10 \mathrm{mM} \mathrm{KCl}, 2 \mathrm{mM}$ DTT, $1 \mathrm{mM}$ PMSF, $1 \mathrm{mM}$ EDTA, $1 \mathrm{mM}$ EGTA, $2 \mathrm{mM}$ sodium orthovanadate, $50 \mathrm{mM} \mathrm{NaF}, 10 \mathrm{mM}$ sodium pyrophosfate, $0.5 \%$ Triton, $5 \mathrm{mg} / \mathrm{ml}$ aprotinin, and $5 \mathrm{mg} / \mathrm{ml}$ leupeptin) and centrifuged at $13000 \mathrm{rpm}$ at $4{ }^{\circ} \mathrm{C}$ for $3 \mathrm{~min}$. The supernatant was used for cytosolic preparation and pellet for nuclear extracts. Cytosolic preparation: the supernatant was saved, centrifuged at $11200 \mathrm{rpm}$ at $4{ }^{\circ} \mathrm{C}$ for $20 \mathrm{~min}$ and the resulting supernatant was used as soluble fraction. Nuclear extracts preparation: the pellet was resuspended in an appropriate volume of ice-cold Buffer C (20 mM Hepes pH 7.5, $400 \mathrm{mM}$ $\mathrm{NaCl}, 1.5 \mathrm{mM} \mathrm{MgCl} 2,10 \mathrm{mM} \mathrm{NaF}, 10 \mu \mathrm{M} \mathrm{Na} \mathrm{MoO}_{4}, 0.1 \mathrm{mM}$ EDTA, $1 \mathrm{mM}$ sodium orthovanadate, $10 \mathrm{mM}$ PNPP, $10 \mathrm{mM}$ $\beta$-glycerophosphate, $20 \%$ glycerol, $2 \mathrm{mM}$ DTT, and protease inhibitors as above) and homogenized. After $30 \mathrm{~min}$ incubation on ice with gently rocking, samples were centrifuged at $13000 \mathrm{rpm}$ at $4^{\circ} \mathrm{C}$ for $10 \mathrm{~min}$ and the resulting supernatant was saved and used as nuclear extract. Protein concentrations in the respective fractions were determined according to the Micro-BCA assay kit (Pierce, Rockford, IL, USA).

Western blotting. Equivalent amounts of protein from the soluble fraction for each sample were resolved by $10 \%$ SDSPAGE, blotted electrophoretically to nitrocellulose membrane, blocked and then incubated overnight at $4{ }^{\circ} \mathrm{C}$ with a rabbit polyclonal antibody that selectively recognized phospho-ERK 1-2 (Cell Signalling Technology Inc., Beverly, MA, USA; diluted $1: 1000$ ) or phospho-CAMKII (Chemicon International, Temecula, CA, USA; diluted 1:750). Bound antibodies were detected with horseradish peroxidaselinked anti-rabbit antibody (Santa Cruz Biotechnology Inc., Santa Cruz, CA, USA; diluted $1: 3000)$ and developed using an enhanced chemiluminescence reagent (Amersham Biosciences, Milan, Italy). To control for protein loading, the membranes were then stripped with T-PBS for $1 \mathrm{~h}$ at $60^{\circ} \mathrm{C}$ and reprobed with a rabbit polyclonal antibody raised against total ERK (Santa Cruz Biotechnology Inc., diluted 1:5000) or total CAMKII (Chemicon International, diluted $1: 1000)$. The relevant immunoreactive bands were quantified with scanning densitometry using Scion Image software. To allow comparison between different autoradiographic films, the density of the bands was expressed as a percentage of the average of control. The value of active phospho-proteins was normalized to the amount of total proteins in the same sample and expressed as a percentage of controls.

PKA activity. The amount of PKA activated in vivo was assayed according to the instructions of the SignaTECT PKA assay system (Promega, Madison, WI, USA). In brief, the isolated brain regions were homogenized in ice-cold buffer containing $0.25 \mathrm{M}$ sucrose, $50 \mathrm{mM}$ Tris- $\mathrm{HCl}$ ( $\mathrm{pH} 7.5)$, $5 \mathrm{mM}$ EGTA, $5 \mathrm{mM}$ EDTA, $1 \mathrm{mM}$ phenylmethylsulfonyl fluoride, $0.1 \mathrm{mM}$ dithiothreitol, $0.1 \%$ Triton $\mathrm{X}-100,10 \mathrm{~g} / \mathrm{ml}$ leupeptin, and $10 \mathrm{~g} / \mathrm{ml}$ aprotinin. The homogenized tissues were briefly centrifuged at $13000 \mathrm{~g}$ at $4^{\circ} \mathrm{C}$, and the supernatant was used as cellular extract. Lysate $(5 \mu \mathrm{l})$ was added to a reaction mixture containing $10 \mu \mathrm{Ci}\left[{ }^{32} \mathrm{P}\right] \mathrm{ATP}, 5 \mu \mathrm{M}$ cAMP, $100 \mu \mathrm{M}$ PKA biotinylated peptide substrate, $40 \mathrm{mM}$ Tris- $\mathrm{HCl}(\mathrm{pH} 7.4), 25 \mathrm{mM} \mathrm{MgCl}_{2}$, and $100 \mu \mathrm{g} / \mathrm{ml}$ bovine serum albumin. Each reaction was immediately incubated at $30^{\circ} \mathrm{C}$ for $5 \mathrm{~min}$ and then terminated by adding $12.5 \mu \mathrm{l}$ of $7.5 \mathrm{M}$ guanidine hydrochloride. An aliquot of the terminated reactions was spotted onto a phosphocellulose filter. After the filters were washed with $2 \mathrm{M} \mathrm{NaCl}$ in $1 \% \mathrm{H}_{3} \mathrm{PO}_{4}$, and deionized water, respectively, the amount of ${ }^{32} \mathrm{P}$-labeled substrate in the filter was measured by scintillation counting.

Transcription factors evaluation. The level of activated transcription factors was measured in the nuclear extracts using transcription factor assay kits (Active Motif, Rixensart, Belgium) based on ELISA method to detect and quantify pCREB and c-Fos activation. Briefly, oligonucleotides containing a cAMP-responsive element (CRE) or a TPA-responsive element (TRE) have been immobilized in a 96-well plate. CREB or cFos contained in nuclear cell extracts bind specifically to these oligonucleotides. By using an antibody directed against phosphorylated CREB or c-Fos, the equivalent transcription factors bound to the oligonucleotide are detected. Addition of a secondary antibody conjugated to horseradish peroxidase provides sensitive colorimetric readout that is quantified by spectrophotometry at $450 \mathrm{~nm}$.

\section{Statistical Analysis}

Data were expressed as mean \pm SEM and analyzed with oneway ANOVA or Kruskal-Wallis test followed by post hoc Tukey's or Dunnett's test. A $p<0.05$ was considered to be significant throughout. All statistical calculations were carried out using Prism, version 4 software (GraphPad, San Diego, CA, USA).

\section{RESULTS}

\section{Behavioral Findings}

Figure 1 shows the differences between groups with respect to the percentage of EPM parameters at all THC doses, administered $30 \mathrm{~min}$ before the test. One-way ANOVA indicated a significant effect of treatment on the percentage of open-arm entries $\left(\mathrm{F}_{(6,63)}=14.34, p<0.0001\right)$, closed-arm entries $\left(\mathrm{F}_{(6,63)}=18.26, p<0.0001\right)$, open-arm time $\left(\mathrm{F}_{(6,63)}=8.01, \quad p<0.001\right)$, and closed-arm time $\left(\mathrm{F}_{(6,63)}=6.58, p=0.0003\right)$. Post hoc analysis found significant differences between THC-treated groups and the vehicle group for doses between 0.075 and $1.5 \mathrm{mg} / \mathrm{kg}$. The anxiolytic effect of THC was dose dependent in the range between 0.015 and $0.75 \mathrm{mg} / \mathrm{kg}\left(r_{\text {open-arm entries }}^{2}=\right.$ $\left.0.95, r_{\text {open-arm time }}^{2}=0.96, p<0.02\right)$. The maximal anxiolytic effect was obtained with the dose of $0.75 \mathrm{mg} / \mathrm{kg}$. The highest dose $(3 \mathrm{mg} / \mathrm{kg})$ was ineffective.

The effect of the $\mathrm{CB} 1$ receptor antagonist AM251 given alone or with THC $(0.75 \mathrm{mg} / \mathrm{kg})$ is reported in Figure 2 . One-way ANOVA showed that treatment significantly affected the percentage of open-arm entries $\left(\mathrm{F}_{(3,36)}=\right.$ 

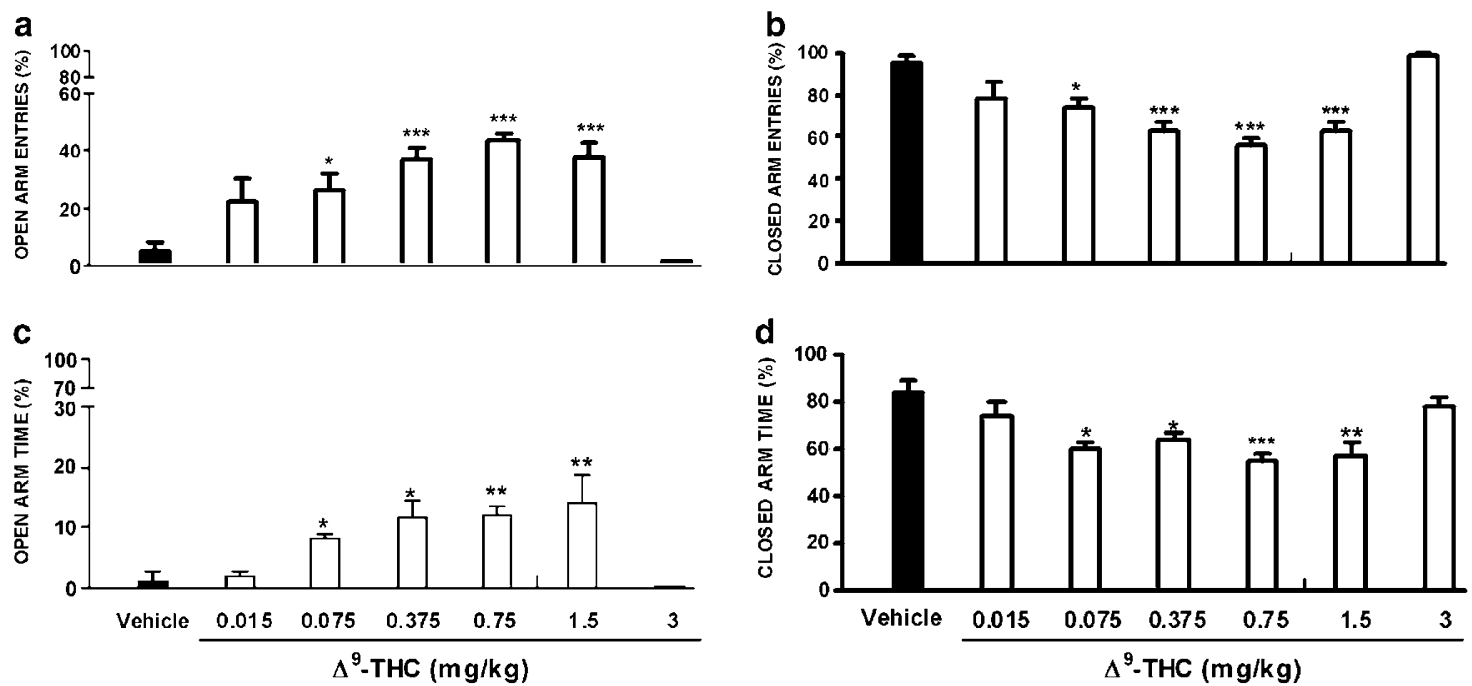

Figure I Effect of systemic THC on behavior in the elevated plus maze (EPM). Male adult Sprague-Dawley rats were injected with vehicle or THC $(0.015-3 \mathrm{mg} / \mathrm{kg}$, i.p.) $30 \mathrm{~min}$ before testing on the EPM for $5 \mathrm{~min}$. Percentage of entries into open (a) and closed (b) arms, percentage of time spent in the open (c) and closed (d) arms. Each column represents the mean \pm SEM of 10 animals. $* p<0.05, * * P<0.01$, **** $P<0.00$ I compared to vehicle group (one-way ANOVA followed by Tukey's test).
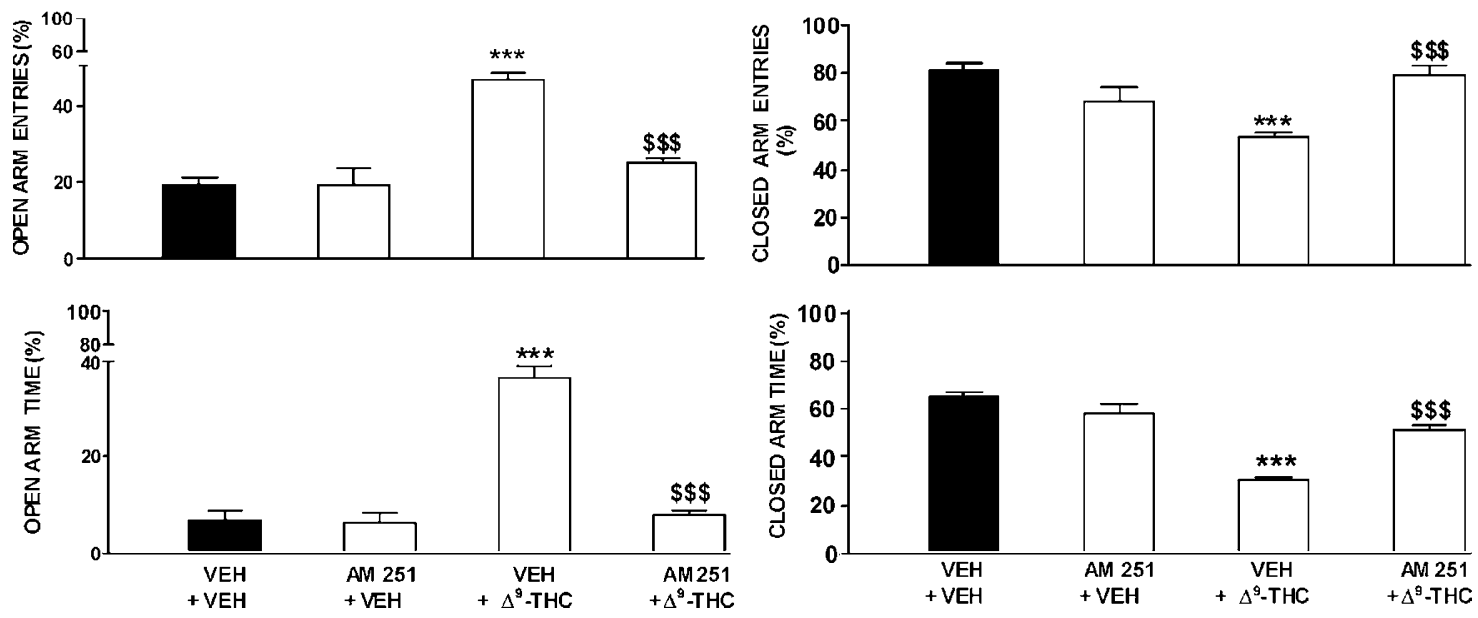

Figure 2 Effect of the $C B$ I cannabinoid receptor antagonist, $A M 25$ I, on THC-induced anxiolytic effects in the EPM. AM25I was given i.p. at a dose of $3 \mathrm{mg} / \mathrm{kg}, 10 \mathrm{~min}$ before THC $(0.75 \mathrm{mg} / \mathrm{kg}$, i.p.), which was given $30 \mathrm{~min}$ before the test. Each column represents the mean \pm SEM of 10 animals. $\mathrm{VEH}=$ vehicle. ${ }^{* * * *} \mathrm{p}<0.00$ I compared to vehicle and AM25I alone; ${ }^{\$ \$ \$} p<0.00$ I compared to THC alone (one-way ANOVA followed by Tukey's test).

$26.06, p<0.0001)$, closed-arm entries $\left(\mathrm{F}_{(3,36)}=9.82, p<0.0001\right)$, open-arm time $\left(\mathrm{F}_{(3,36)}=68.52, p<0.0001\right)$, and closed-arm time $\left(\mathrm{F}_{(3,36)}=35.17, \quad p<0.0001\right)$. Post hoc analysis showed that the cannabinoid antagonist per se did not change emotional reactivity. Pretreatment with AM251 completely blocked the effect of THC on the EPM parameters.

Figure 3 illustrates the effect of the maximal anxiolytic and the highest dose of THC, in the EPM, on spontaneous motor activity. One-way ANOVA indicated a significant effect of drug treatment on horizontal $\left(\mathrm{F}_{(2,27)}=6.47\right.$, $p=0.005)$ and vertical counts $\left(\mathrm{F}_{(2,27)}=6.56, p=0.004\right)$. Post hoc comparison showed that spontaneous motor activity was not affected by the maximal anxiolytic dose of THC. The highest dose significantly reduced both the horizontal and vertical counts.

\section{Biochemical Findings}

All the biochemical experiments were carried out with the THC dose that had maximal anxiolytic effects, that is $0.75 \mathrm{mg} / \mathrm{kg}$.

First of all we checked whether cFos expression was altered in rats exposed to the EPM compared to naïve animals. As shown in Figure 4, cFos levels were significantly higher in the prefrontal cortex $\left(\mathrm{F}_{(4,32)}=4.259, p<0.01\right.$; $p<0.05$ for post hoc comparison between naïve and vehicle) and amygdala $\left(\mathrm{F}_{(4,30)}=6.363, p<0.001 ; p<0.05\right.$ for post hoc comparison between naïve and vehicle) of EPM-tested rats. THC significantly reduced the amounts of cFos in the prefrontal cortex $\left(\mathrm{F}_{(4,32)}=4.259, p<0.01 ; p<0.01\right.$ for post hoc comparison between vehicle and THC) and amygdala $\left(\mathrm{F}_{(4,30)}=6.363, p<0.001 ; p<0.01\right.$ for post hoc comparison 


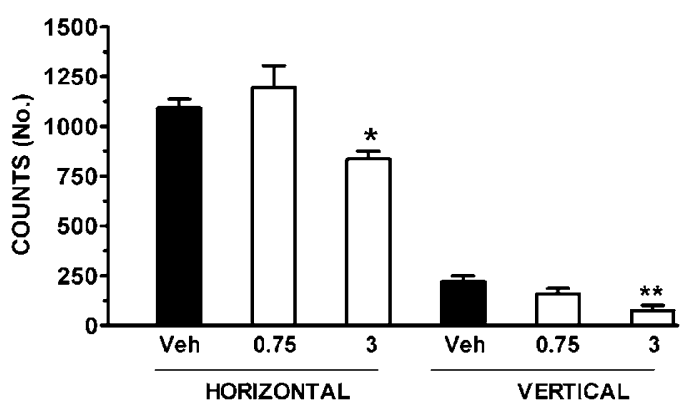

Figure 3 Effect of THC ( 0.75 and $3 \mathrm{mg} / \mathrm{kg}$, i.p.), given $30 \mathrm{~min}$ before the test, on spontaneous motor activity evaluated in terms of horizontal and vertical counts. Each column represents the mean \pm SEM of 10 animals. $\mathrm{VEH}=$ vehicle. ${ }^{*} p<0.05$, $* * 0<0.01$ compared to corresponding vehicle (one-way ANOVA followed by Tukey's test).

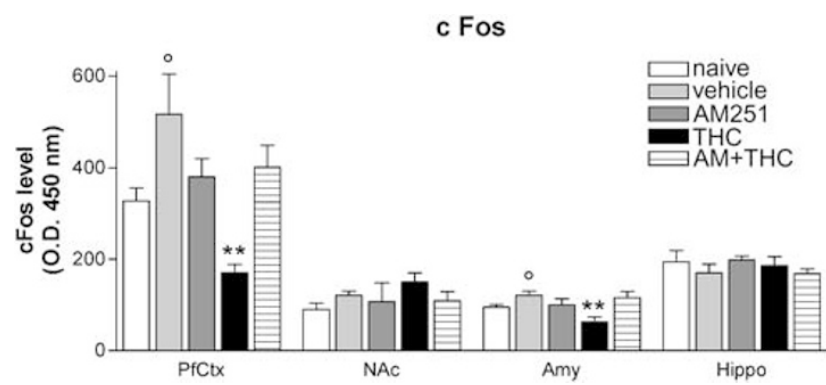

Figure 4 Effect of THC $(0.75 \mathrm{mg} / \mathrm{kg}$ ip) and AM25I pretreatment ( $3 \mathrm{mg} /$ kg i.p., 10 min before THC) on the cFos protein level in the nuclear extracts of different brain areas. cFos in naive animals is also reported. The results are expressed as optical density and are the mean \pm SEM of at least four animals. PfCtx, prefrontal cortex; NAc, nucleus accumbens; Amy, amygdala; Hippo, hippocampus. ${ }^{\circ} p<0.05$ vs naïve; ${ }^{*} * 00.01$ vs vehicle (one-way ANOVA followed by Tukey's test).

between vehicle and THC) without affecting the other cerebral areas. When AM251 was injected before THC, the decrease was reversed, suggesting the effect was CB1 dependent.

Figure 5 shows the levels of phosphorylated CREB in the different rat cerebral areas. As for cFos experiments, first of all we checked whether PCREB level was altered in rats exposed to the EPM compared to naïve animals. No significant alterations were observed between the two groups. In rats treated with THC, only the prefrontal cortex and hippocampus showed a significant increase in the level of phosphorylated CREB. The raise was about $90 \%$ in the prefrontal cortex $\left(\mathrm{F}_{(4,23)}=11.85, p<0.0001 ; p<0.001\right.$ for post hoc comparison between vehicle and THC) and $75 \%$ in the hippocampus $\left(\mathrm{F}_{(4,28)}=3.125, p<0.05 ; p<0.05\right.$ for post hoc comparison between vehicle and THC). AM251 pretreatment fully reversed the increase, indicating that CB1 receptor stimulation was involved.

We next studied the cellular events involved in CREB regulation that could be triggered by $\mathrm{CB} 1$ receptor, studying the activity of PKA, CAMKII, and ERK in the prefrontal cortex and hippocampus of rats treated with THC and then exposed to EPM. As for all the other biochemical experiments, we checked these pathways in the naïve group too. EPM exposure per se did not modify PKA activity in the two brain areas, and THC administration did not alter it (Figure 6).

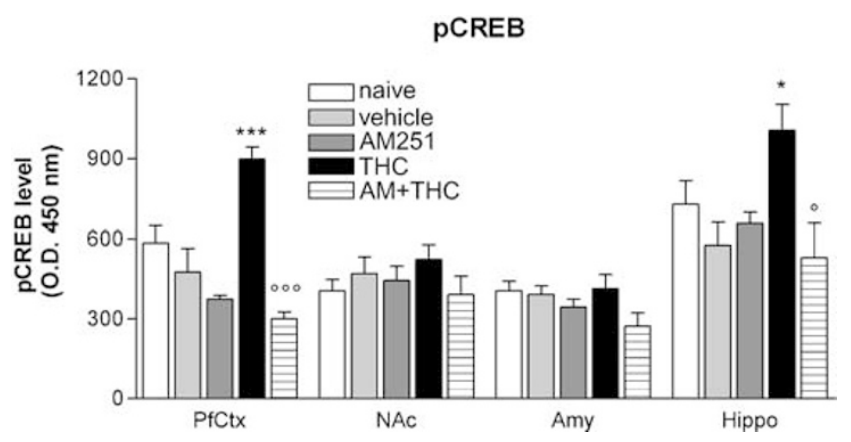

Figure 5 Effect of $\mathrm{THC}(0.75 \mathrm{mg} / \mathrm{kg}$ i.p. $)$ and $\mathrm{AM} 25 \mathrm{I}$ pretreatment ( $3 \mathrm{mg} / \mathrm{kg}$ i.p., $10 \mathrm{~min}$ before THC) on phosphorylated CREB protein level in the nuclear extracts of different brain areas. PCREB in naive animals is also reported. The results are expressed as optical density and are the mean \pm SEM of at least four animals. PfCtx, prefrontal cortex; NAc, nucleus accumbens; Amy, amygdala; Hippo, hippocampus. $* p<0.05$; $* * * * 0.001$ vs vehicle ${ }^{\circ} p<0.05$; ${ }^{*} * * * 0.00$ I vs vehicle ${ }^{\circ} p<0.05 ;{ }^{\circ 00} p<0.00$ I THC (one-way ANOVA followed by Tukey's test).

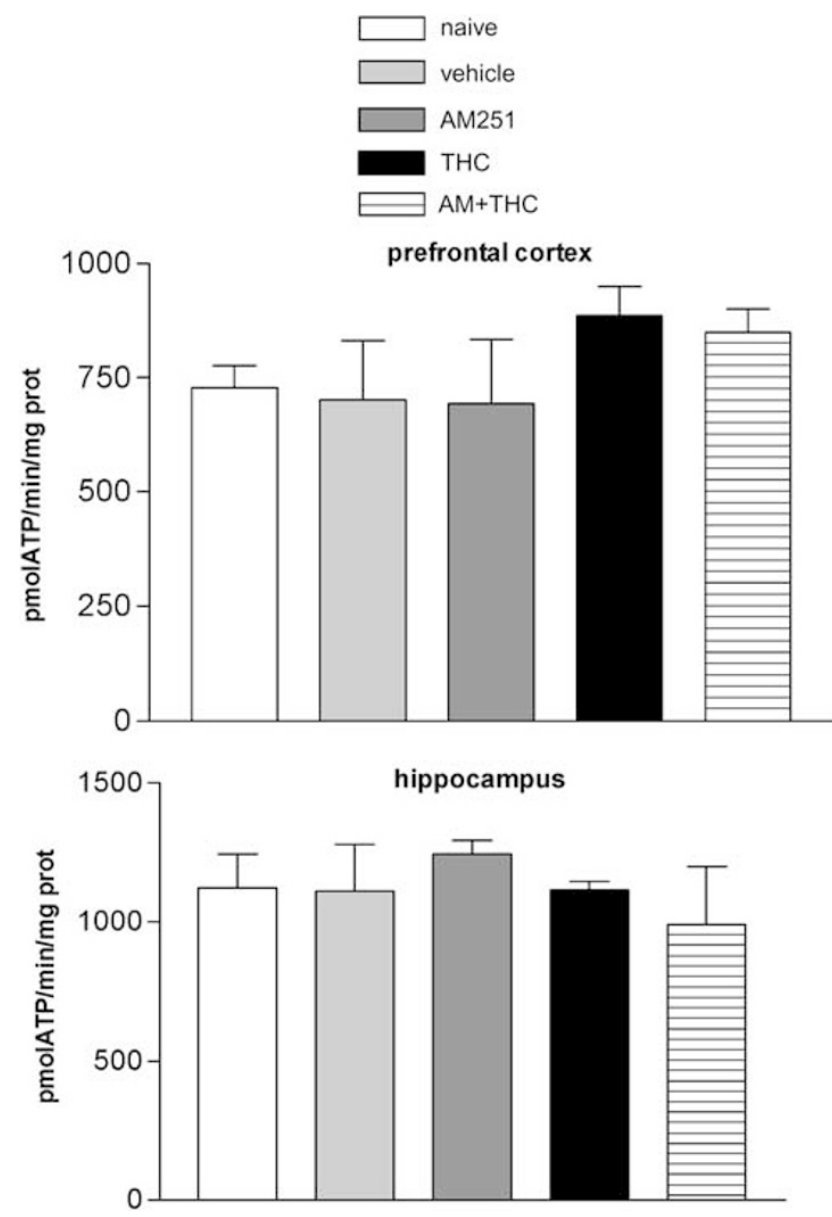

Figure 6 Effect of $\mathrm{THC}(0.75 \mathrm{mg} / \mathrm{kg}$ i.p.) and $\mathrm{AM} 25 \mathrm{I}$ pretreatment ( $3 \mathrm{mg} / \mathrm{kg}$ i.p., $10 \mathrm{~min}$ before THC) on PKA activity in the prefrontal cortex and hippocampus. PKA activity in naive animals is also reported. The results are expressed as pmol ATP/min/mg protein and are the mean \pm SEM of at least four animals.

EPM exposure per se induced a significant reduction in pCAMKII immunoreactivity in the prefrontal cortex $\left(\mathrm{F}_{(4,15)}=9.982, p<0.001 ; p<0.01\right.$ for post hoc comparison between naïve and vehicle), and THC administration did 
a

prefrontal cortex
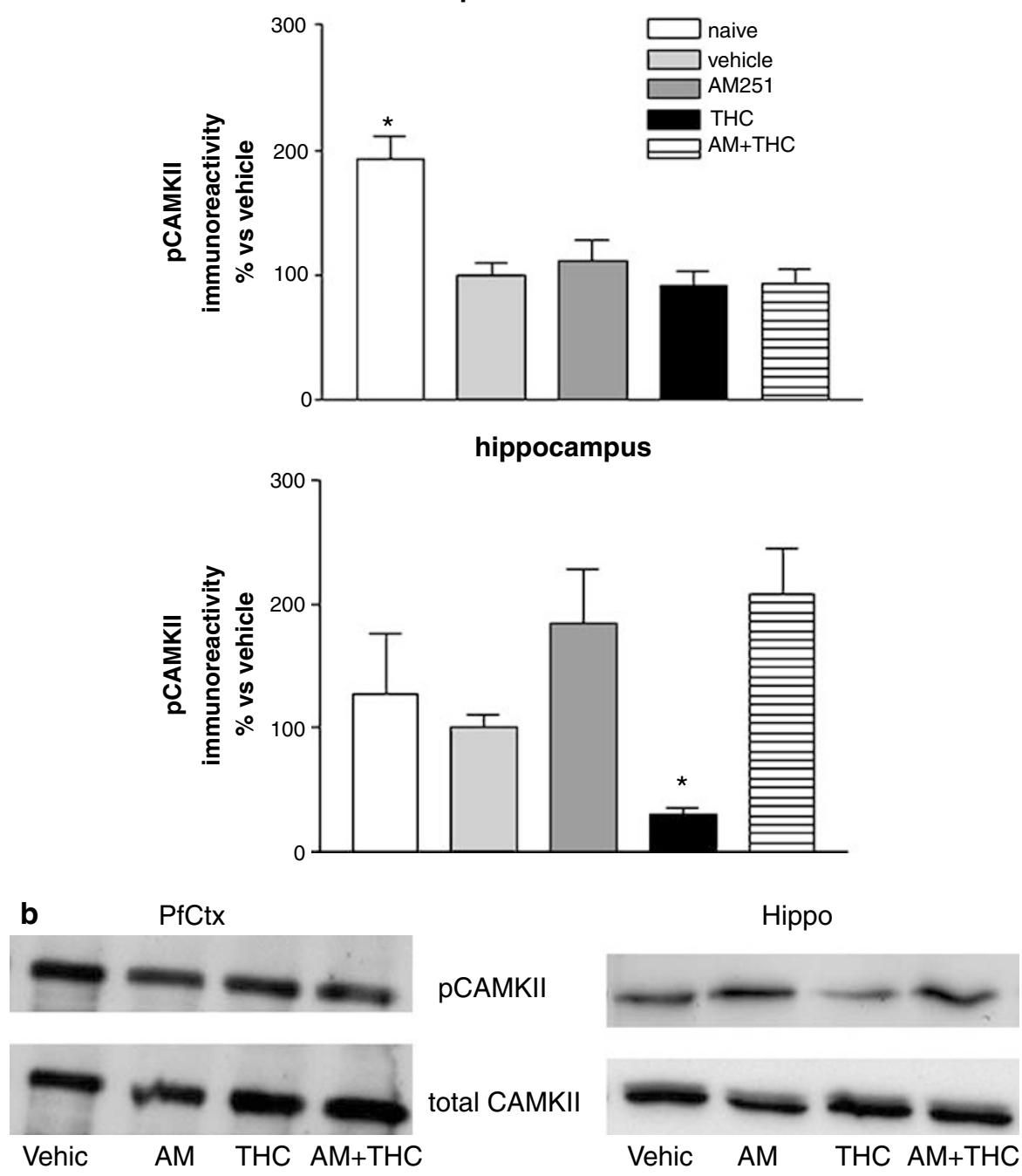

Figure 7 Effect of THC $(0.75 \mathrm{mg} / \mathrm{kg}$ i.p.) and AM25I pretreatment $(3 \mathrm{mg} / \mathrm{kg}$ i.p., $10 \mathrm{~min}$ before THC) on CAMKIl activation in the prefrontal cortex (PfCtx) and hippocampus (Hippo). CAMKIl activation in naive animals is also reported. (a) Densitometric analysis; (b) representative autoradiograms. The results are expressed as the percentage pCAMKII immunoreactivity vs vehicle and are the mean \pm SEM of at least three animals. $* 2<0.05$ vs vehicle (one-way ANOVA followed by Tukey's test).

not modify further this picture. In the hippocampus, exposure to EPM did not modify the level of phosphoCAMKII, THC instead, significantly lowered it $\left(\mathrm{F}_{(4,15)}=\right.$ 4.354, $p<0.05 ; p<0.05$ for post hoc comparison between vehicle and THC) in a CB1-dependent manner, as it was fully reversed by the AM251 pretreatment (Figure 7).

Concerning pERK immunoreactivity in the prefrontal cortex, EPM exposure per se induced a trend to increase pERK levels, but it did not reach the statistical significance (Figure 8). THC treatment produced an increase in pERK immunoreactivity significantly different from that induced by EPM $\left(\mathrm{F}_{(4,11)}=7.110, p<0.01 ; p<0.05\right.$ for post hoc comparison between vehicle and THC) which was prevented by the CB1 antagonist (Figure 8). When the same analysis were performed in the hippocampus, no significant changes were observed (Figure 8).

The causative link between the ERK pathway and THC anxiolytic effect was tested in rats by microinjecting in the prefrontal cortex SL327, a drug that prevents the activation of ERK by inhibiting MEK, the upstream kinase of ERK
(Atkins et al, 1998; Rubino et al, 2005). SL327 microinjection $(30 \mu \mathrm{g})$ lowered ERK phosphorylation in rats prefrontal cortex $(p<0.01$, Student's $t$-test; Figure 9a). When injected 5 min before THC, it prevented THC anxiolytic effect both in term of percentage of open-arm time $\left(\mathrm{F}_{(3,11)}=7.189\right.$, $p<0.01 ; p<0.01$ for post hoc comparison between THC and SL327+THC; Figure 9b) and open-arm entries $\left(\mathrm{F}_{(3,11)}=11.0, p<0.01 ; p<0.001\right.$ for post hoc comparison between THC and SL327 + THC; Figure 9b). Finally, SL327 pretreatment fully reversed THC-induced CREB activation in the prefrontal cortex $\left(\mathrm{F}_{(3,18)}=18.14, p<0.0001 ; p<0.001\right.$ for post hoc comparison between THC and SL327 + THC; Figure 9c).

\section{DISCUSSION}

The present study provides evidence of a dose-dependent anxiolytic effect of THC obtained at doses between 0.075 and $1.5 \mathrm{mg} / \mathrm{kg}, 0.75 \mathrm{mg} / \mathrm{kg}$ being the most effective. The lack 

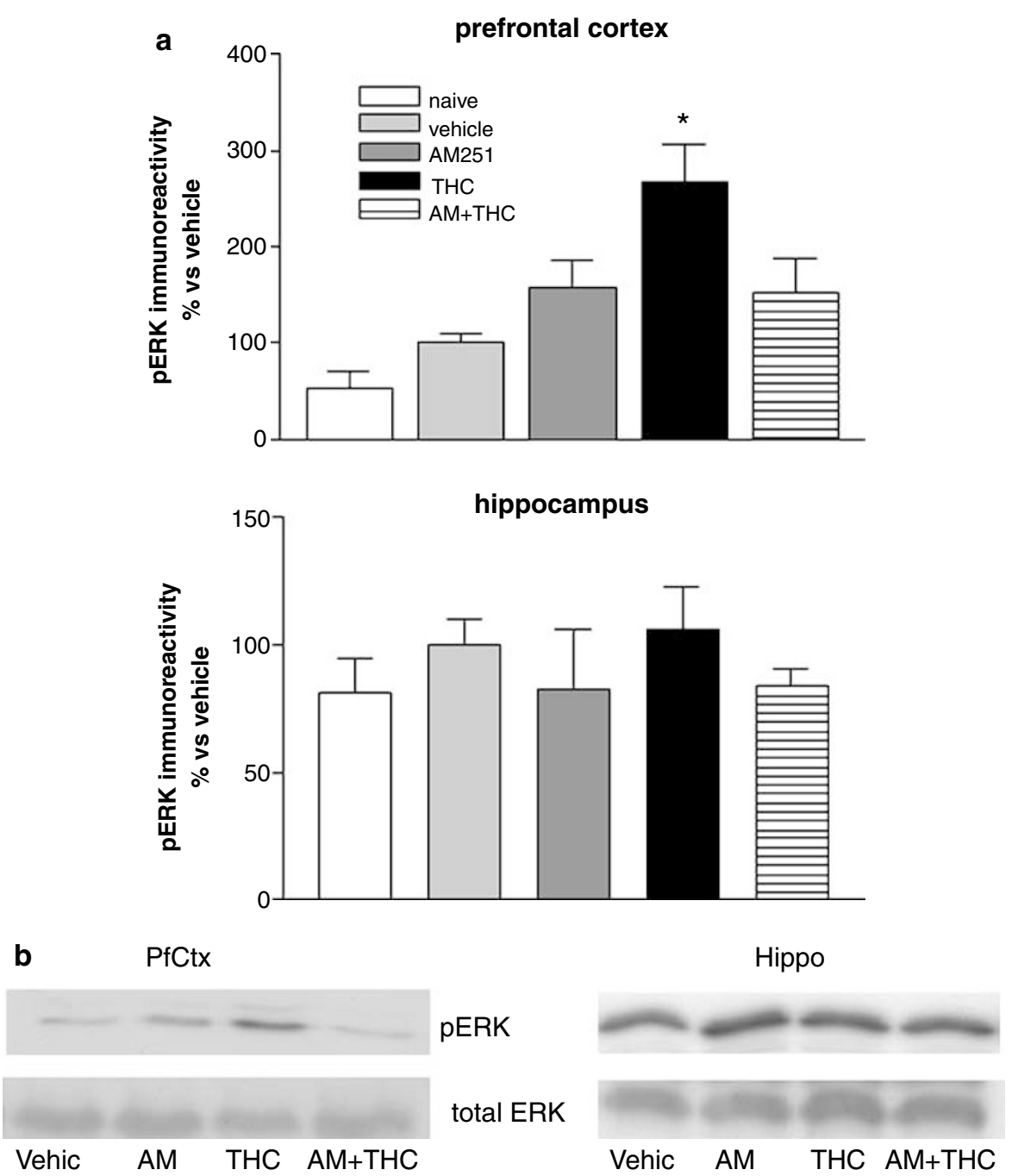

Figure 8 Effect of THC $(0.75 \mathrm{mg} / \mathrm{kg}$ i.p.) and AM25I pretreatment ( $3 \mathrm{mg} / \mathrm{kg}$ i.p., $10 \mathrm{~min}$ before THC) on ERK activation in the prefrontal cortex (PfCtx) and hippocampus (Hippo). ERK activation in naive animals is also reported. (a) Densitometric analysis; (b) representative autoradiograms. The results are expressed as the percentage pERK immunoreactivity vs vehicle and are the mean \pm SEM of at least three animals. $* p<0.05$ vs vehicle (one-way ANOVA followed by Tukey's test).

of anxiolytic effect with the highest dose $(3 \mathrm{mg} / \mathrm{kg})$ may be explained by the motor impairment which could have interfered with the behavior used to measure anxiolytic effects.

Our findings agree with Berrendero and Maldonado (2002) who found an anxiolytic effect of THC in mice, at a dose of $0.3 \mathrm{mg} / \mathrm{kg}$, using the light-dark box. Onaivi et al (1990) using the EPM found either an anxiogenic or no effect of THC using a range of doses higher than ours. Previous studies have shown that cannabinoid agonists have a dose-dependent biphasic effect in anxiety-like responses (Onaivi et al, 1990; Rodriguez de Fonseca et al, 1996; Valjent et al, 2002), as already observed for other pharmacological actions induced by cannabinoids (Sanudo-Pena et al, 2000; Sulcova et al, 1998; Braida et al, 2004).

The CB1 antagonist AM251 reversed the anxiolytic-like effect of THC, providing evidence of the specific involvement of CB1 cannabinoid receptors in this behavior.

While we were drafting this manuscript, the paper of Patel and Hillard (2006) appeared reporting different results after injection of THC in mice. THC $(0.25-10 \mathrm{mg} / \mathrm{kg}$ i.p. $)$ did not produce anxiolytic-like response in the EPM. This disagrees with our data but the different sensitivity to THC might be due to species differences (mouse $v s$ rat).

However, the most original finding of the present study concerns the neuroanatomical and cellular correlates of THC's anxiolytic effect. First of all using c-Fos strategy to map neural circuitries underlying behavioral responses, we saw that the prefrontal cortex and the amygdala were the main cerebral regions involved in the anxiolytic effect. In both areas, we found a significant decrease in cFos activation, suggesting neural deactivation that was fully reversed by pretreatment with AM251, thus indicating the CB1 dependence of this effect.

The responsiveness of these brain regions is not surprising since the prefrontal cortex and the amygdala are anatomically connected and strongly implicated in anxiety mechanisms. Activation of the amygdala results in behavioral and physiological responses associated with anxiety and panic-like behavior, whereas lesions reduce 


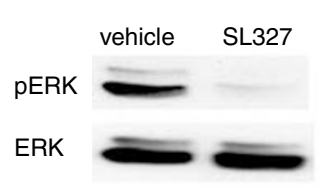

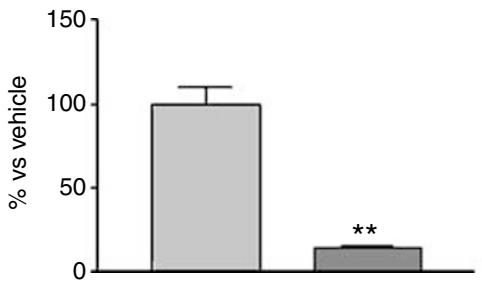
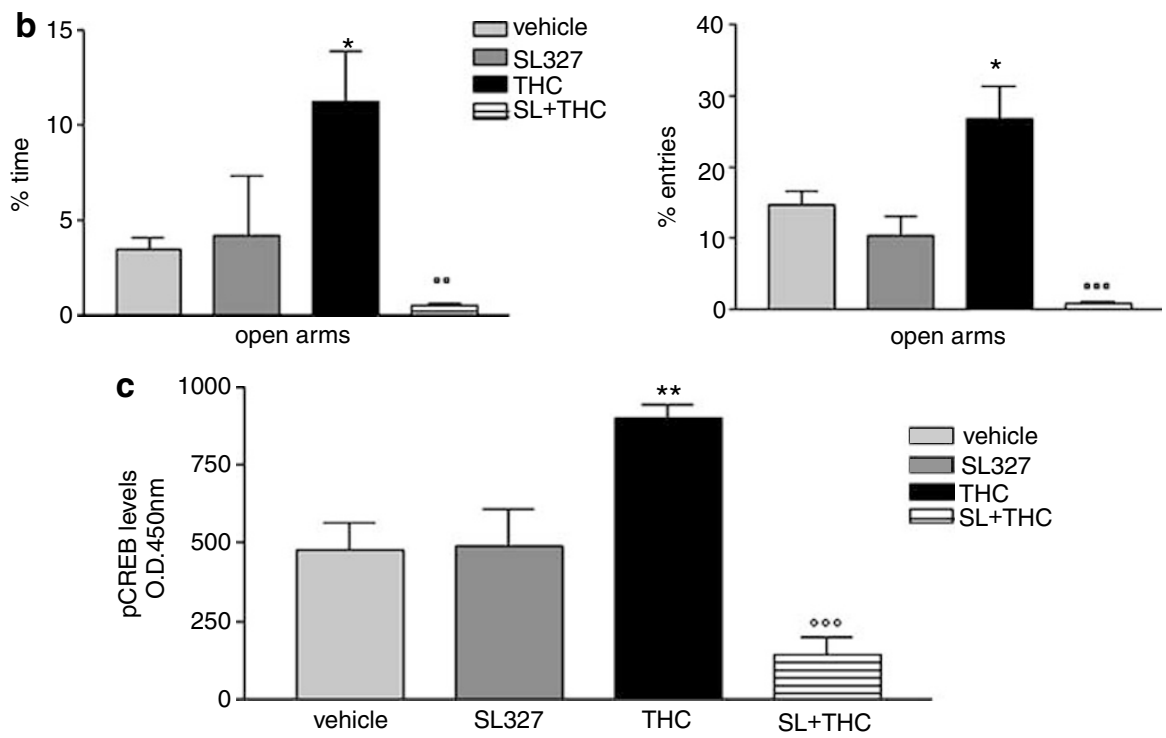

Figure 9 (a) Effect of SL327 microinjection $(30 \mu \mathrm{g})$ in the prefrontal cortex on p-ERK immunoreactivity. $* * * 2<0.0$ I vs vehicle (Student's t-test). (b) Effect of SL327 pretreatment ( $30 \mu \mathrm{g}$ in the prefrontal cortex, 5 min before THC) on THC anxiolytic effect evaluated in the EPM. * $p<0.05$ vs vehicle; ${ }^{\circ 0} p<0.0$ I, ${ }^{000} \mathrm{p}<0.00 \mathrm{I}$ vs THC alone (one-way ANOVA followed by Tukey's test). (c) Effect of SL 327 pretreatment ( $30 \mu \mathrm{g}$ in the prefrontal cortex, 5 min before THC) on THC-induced CREB phosphorylation in the prefrontal cortex. ${ }^{*} *<0.01$ vs vehicle; ${ }^{\circ 00} p<0.00$ I vs THC alone (one-way ANOVA followed by Tukey's test).

such effects (for a review, see Phelps and LeDoux, 2005). Along similar lines, parts of the prefrontal cortex have been reported to influence the induction of anxiety and fearrelated behavior and may play a role in autonomic reactions during stress and anxiety (Davidson, 2002). Interestingly, both the prefrontal cortex and the amygdala, where we found less cFos activation, can be activated by environmentally evoked anxiety (for a review, see Kovacs, 1998). Likewise, we found that vehicle-treated animals exposed to the EPM had higher levels of cFos in these brain regions than rats handled in the same manner but not subjected to the test (naïve). This is in agreement with Hinks et al (1996) and Duncan et al (1996) who reported similar increases in cFos expression, respectively in the amygdala and in the medial prefrontal areas after exposure to the EPM. In a similar stressful situation that induces anxiety in rats, that is exposure to cat odor, benzodiazepines had an anxiolytic effect that was paralleled by reduction in cFos expression in the medial prefrontal cortex and-though to a lesser extent - in the amygdala (McGregor et al, 2004).

Therefore, as already proposed in McGregor's paper for midazolam or more in general for benzodiazepines (McNaughton and Gray, 2000), we suggest that THC disinhibits the approach in situations of fear or uncertainty and promotes risk assessment behavior at the expense of flight. This shift in defensive behavior involves action on different brain regions: the prefrontal cortex may play a key role in coping with stress, and THC can deactivate this stress-related circuitry, whereas THC's effect in the amygdala might reflect specific action on the unconditioned fear response. Therefore, we can speculate that THC's anxiolytic effect may be at least partially due to prevention of the activation of an anxiety circuit involving the prefrontal cortex and amygdala.

The second result worth commenting is the association of THC's anxiolytic effect with increased CREB activation and related intracellular pathways in the prefrontal cortex and hippocampus. As for cFos experiments, we first checked the effect of EPM per se on these biochemical parameters. In the hippocampus neither phospho-CREB nor PKA, pCAMKII, and pERK levels were altered by EPM exposure. THC injection instead, significantly increased pCREB along with a decrease in the activity of CAMKII. As this kinase has an inhibitory effect on CREB activation, its reduction accounts for the increased pCREB levels triggered by THC through a CB1-dependent mechanism.

In the prefrontal cortex EPM exposure per se did not alter CREB activity, however it significantly reduced pCAMKII contents and provoked a trend to increase in pERK. The injection of THC instead increased pCREB level, did not induce further changes in pCAMKII other than those provoked by EPM exposure, and produced an increase in ERK activation that resulted more sustained and significantly different from that induced by EPM per se. On this 
basis, we suggest that in the prefrontal cortex THC's anxiolytic effect is associated with $\mathrm{CB} 1$ receptor activation triggering CREB phosphorylation via ERK cascade. This picture is also supported by the experiments with SL327. The selective block of ERK activation in the prefrontal cortex fully prevented both THC-induced increase in CREB phosphorylation and THC's anxiolytic effect, highlighting the causative link between these events.

To our knowledge, this is the first study aimed at finding a neural correlate of THC's anxiolytic effect and it indicates that CREB is an important player. In line with our results, Barrot et al (2005) reported that in a model of protracted social isolation in adult rats, where they observed increased anxiety-like behavior, CREB activity was reduced in the nucleus accumbens. Likewise, Pandey et al (2005) found that lowered CREB function in the amygdala was associated with anxiety-like behavior in alcohol preferring rats, and increasing CREB phosphorylation in these animals relieved anxiety.

Taken together these results suggest that CREB is a key regulator of the reactivity of brain circuits and could therefore influence individual sensitivity to emotional stimuli. However, as a transcription factor, CREB itself is unlikely to be the molecule acutely affecting behavior. Rather, changes in CREB activity could alter the expression of specific gene products involved in the modulation of anxiety such as neuropeptide $\mathrm{Y}$ and brain-derived neurotrophic factor (Heilig, 2004; Branchi et al, 2006). This hypothesis requires further research.

Lastly, we did not find any alteration in CREB activation in the amygdala, where cFos levels were lowered, as they were in the prefrontal cortex. This lack of activation might be explained by some indirect effect of THC in this area rather than a direct one (ie not triggered by the stimulation of $\mathrm{CB} 1$ receptors in the amygdala). Consistent with this notion, there are extensive reciprocal connections between the amygdala and prefrontal cortex (Amaral et al, 1992) and the prefrontal cortex inputs excite neurons in the amygdala (Likhtik et al, 2005).

On this basis we might suggest that the THC-induced reduction in neural activation in the prefrontal cortex might also lead to neural deactivation in the amygdala. On the other hand, a direct effect of THC in the amygdala also cannot be ruled out, as suggested by the presence of CB1 receptors in this brain region. If this is the case, the intracellular pathway linked to CB1 receptor stimulation is still unclear but it does not involve CREB activation.

In conclusion, this study casts fresh light on the neurochemical and neuroanatomical substrates of THC's anxiolytic effect and highlights the importance of the cannabinoid system in these behaviors. The stimulation of CB1 receptors in the prefrontal cortex, amygdala and hippocampus, with the subsequent activation of different signaling pathways, might be the initial event underlying the effects of the cannabinoids on anxious states.

\section{ACKNOWLEDGEMENTS}

This work was supported by Ministero dell'Istruzione, dell'Università e della Ricerca PRIN2004, and by University of Insubria FAR2005. We are grateful to Judy Baggot for language editing of the manuscript.

\section{REFERENCES}

Amaral DG, Price JL, Pitkanen A, Carmichael ST (1992). Anatomical organization of the primate amygdaloid complex. In: Aggleton JP (ed). The Amygdala: Neurobiological Aspects of Emotion, Memory and Mental Dysfunction. Wiley-Liss: New York. pp 1-66.

Arevalo C, de Miguel R, Hernandez-Tristan R (2001). Cannabinoid effects on anxiety-related behaviours and hypothalamic neurotransmitters. Pharmacol Biochem Behav 70: 123-131.

Atkins CM, Selcher JC, Petraitis JJ, Trzaskos JM, Sweatt JD (1998). The MAPK cascade is required for mammalian associative learning. Nat Neurosci 1: 602-609.

Barrot M, Olivier JD, Perrotti LI, DiLeone RJ, Berton O, Eisch AJ et al (2002). CREB activity in the nucleus accumbens shell controls gating of behavioral responses to emotional stimuli. Proc Natl Acad Sci USA 99: 11435-11440.

Barrot M, Wallace DL, Bolanos CA, Graham DL, Perrotti LI, Neve RL et al (2005). Regulation of anxiety and initiation of sexual behavior by CREB in the nucleus accumbens. Proc Natl Acad Sci USA 102: 8357-8362.

Berrendero F, Maldonado R (2002). Involvement of the opioid system in the anxiolytic-like effects induced by $\Delta^{9}$-tetrahydrocannabinol. Psychopharmacology 163: 111-117.

Bortolato M, Campolongo P, Mangieri RA, Scattoni ML, Frau R, Trezza V et al (2006). Anxiolytic-like properties of the anandamine transport. Inhibitor AM404. Neuropsycopharmaco$\log y$ 31: 2652-2659.

Braida D, Iosuè S, Pegorini S, Sala M (2004). Delta 9-tetrahydrocannabinol-induced conditioned place preference and intracerebroventricular self-administration in rats. Eur J Pharmacol 506: 63-69.

Braida D, Sala M (2000). Cannabinoid-induced working memory impairment is reversed by a second generation cholinesterase inhibitor in rats. Neuroreport 11: 2025-2029.

Branchi I, D’Andrea I, Sietzema J, Fiore M, Di Fausto V, Aloe L et al (2006). Early social enrichment augments adult hippocampal BDNF levels and survival of BrdU-positive cells while increasing anxiety- and 'depression'-like behavior. J Neurosci Res 83: 965-973.

Carlezon Jr WA, Duman RS, Nestler EJ (2005). The many faces of CREB. Trends Neurosci 28: 436-445.

Davidson RJ (2002). Anxiety and affective style: role of prefrontal cortex and amigdala. Biol Psychiatry 51: 68-80.

Duncan GE, Knapp DJ, Breese GR (1996). Neuroanatomical characterization of c-fos induction in rat behavioral models of anxiety. Brain Res 713: 79-91.

Genn RF, Tucci S, Marco EM, Viveros MP, File SE (2004). Unconditioned and conditioned anxiogenic effects of the cannabinoid receptor agonist CP 55,940 in the social interaction test. Pharmacol Biochem Behav 77: 567-573.

Hampson RE, Evans GJ, Mu J, Zhuang SY, King VC, Childers SR et al (1995). Role of cyclic AMP dependent protein kinase in cannabinoid receptor modulation of potassium 'A-current' in cultured rat hippocampal neurons. Life Sci 56: 2081-2088.

Heffner TG, Hartman JA, Seiden LS (1980). A rapid method for the regional dissection of the rat brain. Pharmacol Biochem Behav 13: $453-456$.

Heilig M (2004). The NPY system in stress, anxiety and depression. Neuropeptides 38: 213-224.

Hinks GL, Brown P, Field M, Poat JA, Hughes J (1996). The anxiolytics CI-988 and chlordiazepoxide fail to reduce immediate early gene mRNA stimulation following exposure to the rat elevated X-maze. Eur J Pharmacol 312: 153-161.

Hogg S (1996). A review of the validity and variability of the elevated plus-maze as an animal model of anxiety. Pharmacol Biochem Behav 54: 21-30. 
Howlett AC, Mukhopadhyay S (2000). Cellular signal transduction by anandamide and 2-arachidonoylglycerol. Chem Phys Lipids 108: $53-70$.

Iversen L (2003). Cannabis and the brain. Brain 126: 1252-1270.

Kathuria S, Gaetani S, Fegley D, Valino F, Duranti A, Tontini A et al (2003). Modulation of anxiety through blockade of anandamide hydrolysis. Nat Med 9: 76-81.

Kovacs KJ (1998). c-Fos as a transcription factor: a stressful (re)view from a functional map. Neurochem Int 33: 287-297.

Likhtik E, Pelletier JG, Paz R, Pare D (2005). Prefrontal control of the amygdala. J Neurosci 25: 7429-7437.

Lister RG (1987). The use of a plus-maze to measure anxiety in the mouse. Psychopharmacology (Berl) 92: 180-185.

Marco EM, Perez-Alvarez L, Borcel E, Rubio M, Guaza C, Ambrosio E et al (2004). Involvement of 5-HT1A receptors in behavioural effects of the cannabinoid receptor agonist CP 55,940 in male rats. Behav Pharmacol 15: 21-27.

Marin S, Marco E, Biscaia M, Fernandez B, Rubio M, Guaza C et al (2003). Involvement of the kappa-opioid receptor in the anxiogenic-like effect of CP 55940 in male rats. Pharmacol Biochem Behav 74: 649-656.

McGregor IS, Hargreaves G, Apfelbach R, Hunt GE (2004). Neuronal correlates of cat odr-induced anxiety in rats: regionspecific effects of the benzodiazepine midazolam. J Neuroscince 24: 4134-4144.

McNaughton N, Gray JA (2000). Anxiolytic action on the behavioural inhibition system implies multiple types of arousal contribute to anxiety. J Affect Disord 61: 161-176.

Onaivi ES, Green MR, Martin BR (1990). Pharmacological characterization of cannabinoids in the elevated plus maze. J Pharmacol Exp Ther 253: 1002-1009.

Pandey SC (2003). Anxiety and alcohol abuse disorders: a common role for CREB and its target, the neuropeptide Y gene. Trends Pharmacol Sci 24: 456-460.

Pandey SC, Zhang H, Roy A, Xu T (2005). Deficits in amygdaloid cAMP-responsive element-binding protein signaling play a role in genetic predisposition to anxiety and alcoholism. J Clin Invest 115: 2762-2773.

Patel S, Hillard CJ (2006). Pharmacological evaluation of cannabinoid receptor ligands in a mouse model of anxiety: further evidence for an anxiolytic role for endogenous cannabinoid signaling. $J$ Pharmacol Exp Ther 318: 304-311.

Phelps EA, LeDoux JE (2005). Contributions of the amygdala to emotion processing: from animal models to human behaviour. Neuron 48: 175-187.

Rodriguez de Fonseca F, Rubio P, Menzaghi F, Merlo-Pich E, Rivier J, Koob GF et al (1996). Corticotropin-releasing factor (CRF) antagonist [D-Phe12,Nle21,38,C alpha MeLeu37] CRF attenuates the acute actions of the highly potent cannabinoid receptor agonist HU-210 on defensive-withdrawal behavior in rats. J Pharmacol Exp Ther 276: 56-64.

Rubino T, Forlani G, Vigano D, Zippel R, Parolaro D (2004). Modulation of extracellular signal-regulated kinases cacade by chronic delta 9-tetrahydrocannabinol treatment. Mol Cell Neurosi 25: 355-362.

Rubino T, Vigano D, Massi P, Spinello M, Zagato E, Gianoni G et al (2000). Chronic delta 9-tetrahydrocannabinol treatment increases cAMP levels and cAMP-dependent protein kinase activity in some rat brain regions. Neuropharmacology 39: 1331-1336.

Rubino T, Forlani G, Vigano D, Zippel R, Parolaro D (2005). Ras/ ERK signalling in cannabinoid tolerance: from behaviour to cellular aspects. J Neurochem 93: 984-991.

Sanudo-Pena MC, Romero J, Seale GE, Fernandez-Ruiz JJ, Walker JM (2000). Activational role of cannabinoids on movement. Eur J Pharmacol 391: 269-274.

Sulcova E, Mechoulam R, Fride E (1998). Biphasic effects of anandamide. Pharmacol Biochem Behav 59: 347-352.

Valjent E, Mitchell JM, Besson MJ, Caboche J, Maldonado R (2002). Behavioural and biochemical evidence for interactions between $\Delta^{9}$ tetrahydrocannabinol and nicotine. Br J Pharmacol 135: 564-578.

Valjent E, Pages C, Rogard M, Besson MJ, Maldonado R, Caboche J (2001). Delta 9-tetrahydrocannabinol-induced MAPK/ERK and Elk-1 activation in vivo depends on dopaminergic transmission. Eur J Neurosci 14: 342-352.

Viveros MP, Marco EM, File SE (2005). Endocannabinoid system and stress and anxiety responses. Pharmacol Biochem Behav 81: 331-342.

Wu X, McMurray CT (2001). Calmodulin kinase II attenuation of gene transcription by preventing cAMP response elementbinding protein (CREB) dimerization and binding of the CREB-binding protein. J Biol Chem 276: 1735-1741. 